\title{
UNIVERSITYOF
}

FORWARD

THINKING

WESTMINSTER ${ }^{\text {用 }}$

WestminsterResearch

http://www.westminster.ac.uk/westminsterresearch

\section{A Wireless Sensor Network for Early Forest Fire Detection and Monitoring as a Decision Factor in the Context of a Complex Integrated Emergency Response System Abdullah, S., Masar, S., Bertalan, S., Coskun, A. and Kale, I.}

This is a copy of the author's accepted version of a paper subsequently published in the proceedings of the IEEE Workshop on Environmental, Energy, and Structural Monitoring Systems, Milan, Italy, 24 to 25 July 2017.

It is available online at:

http://ieeexplore.ieee.org/document/8052688/

(C) 2017 IEEE . Personal use of this material is permitted. Permission from IEEE must be obtained for all other uses, in any current or future media, including reprinting/republishing this material for advertising or promotional purposes, creating new collective works, for resale or redistribution to servers or lists, or reuse of any copyrighted component of this work in other works.

The WestminsterResearch online digital archive at the University of Westminster aims to make the research output of the University available to a wider audience. Copyright and Moral Rights remain with the authors and/or copyright owners.

Whilst further distribution of specific materials from within this archive is forbidden, you may freely distribute the URL of WestminsterResearch: ((http://westminsterresearch.wmin.ac.uk/)).

In case of abuse or copyright appearing without permission e-mail repository@westminster.ac.uk 


\title{
A Wireless Sensor Network for Early Forest Fire Detection and Monitoring as a Decision Factor in the Context of a Complex Integrated Emergency Response System
}

\author{
Sakib Abdullah, Sandor Bertalan, Stanislav Masar, Adem Coskun and Izzet Kale \\ Applied DSP and VLSI Research Group, University of Westminster, London, UK, \\ email: s.abdullah1, s.bertalan, s.masar1, a.coskun, kalei@westminster.ac.uk
}

\begin{abstract}
Wildfires, often dubbed megafires, have in recent times increased in both frequency and scale, owing largely to human error as well as climate change. Due to their uncontrolled unpredictable rapid growth and behaviour, they can quickly become difficult to contain, leading to significant loss of lives, wildlife and property. It is therefore critical to tackle such fires in the early stages. This demands a reduction in the initial time to detection while ensuring reliability and a low false alarm rate. This paper discusses a new category of compact, easily deployable and energy efficient approach to sensor nodes for the continued monitoring of forest environments as well as the early detection of fires in their infancy based on a combination of sensory inputs. The sensor network reported in this paper was tested with other subsystems/technologies, in a real-life firefighting trial as part of a coordinated firefighting scenario with promising results.

Index Terms-Forest Fires, Wireless Sensor Network, Digital Signal Processing, Low Power
\end{abstract}

\section{INTRODUCTION}

The frequency of large-scale forest fires continues to increase aided several factors such as climate change, urbanisation and arson. These resulting Mega-fires have devastating effects on lives, property and the environment. To provide some perspective, research indicates some $80-90 \%$ of wildfires in the United States are deemed to be a result of human activity [1]. To this effect, over the ages, various technologies have been deployed for the monitoring of regions susceptible to such fires. These have ranged from manned watch towers, to advanced cameras as well as satellite imaging. However, these methods are limited by various factors such as the need to build expensive, dedicated infrastructure on what is often challenging terrain or being reliant on visual cues which may not always be easily detected on time. In addressing these challenges, and certainly in keeping up with technology and the advent of the Internet of Things (IoT), there has been a shift towards the use of Wireless Sensor Networks (WSN) in this area.

WSNs afford numerous advantages when it comes to monitoring and detection of wildfires. They can be highly scalable in covering large areas allowing control over node density to align with cost/performance requirements as well as providing better position accuracy in locating potential incidents. In addition, the use of a range of sensors can potentially enable earlier detection of fires before any visual cues manifest. Evolving technology makes possible ever tighter integration of sensors and electronics into a smaller footprint as well as delivering power and cost benefits. These advantages have led to notable developments in the use of WSNs for forest fire detection/monitoring with active deployments in various countries as well as an increasing number of publications in the field. Research in [2] discusses a forest fire surveillance system with WSNs that employs the common two level hierarchy comprised of sensor nodes and data sinks. All nodes are based on a low power 16-bit microcontroller as well as temperature, humidity and light intensity sensors, where the aspects of network lifetime, power autonomy and harvesting are not discussed. The network presented in [3] is comprised of a single type of node based on temperature, humidity and $\mathrm{CO} 2$ sensors, that keeps track of allowable thresholds on monitored parameters and dynamically adjusts the frequency of readings. In terms of network longevity, an intelligent sleep solution is opted as opposed to exploring energy harvesting techniques. In a more recent effort [4], the authors discuss a WSN composed of a combination of central and standard sensor nodes. A range of parameters such as temperature, humidity, $\mathrm{CO}, \mathrm{CO} 2$, precipitation and wind speed and direction are captured on central nodes. On the power autonomy front only the central nodes designs are equipped with power harvesting facility. The network presented in [5] is composed of sensor motes which relay data back to a base station for eventual upload to a server for client access. The motes were based on off-theshelf technology. Sensory data collected by this system was limited to temperature, humidity and atmospheric pressure. Other points of interest are the availability of positioning information and that the motes were powered by standard batteries.

The work reported in this paper was undertaken as a part of the Advanced Fire Fighting (AF3) Project that operates under the 7th Framework Programme (FP7), the European Unions Research and Innovation funding programme. The project consolidates the expertise and technologies of the various partners involved, in delivering a seamless and complete forest 


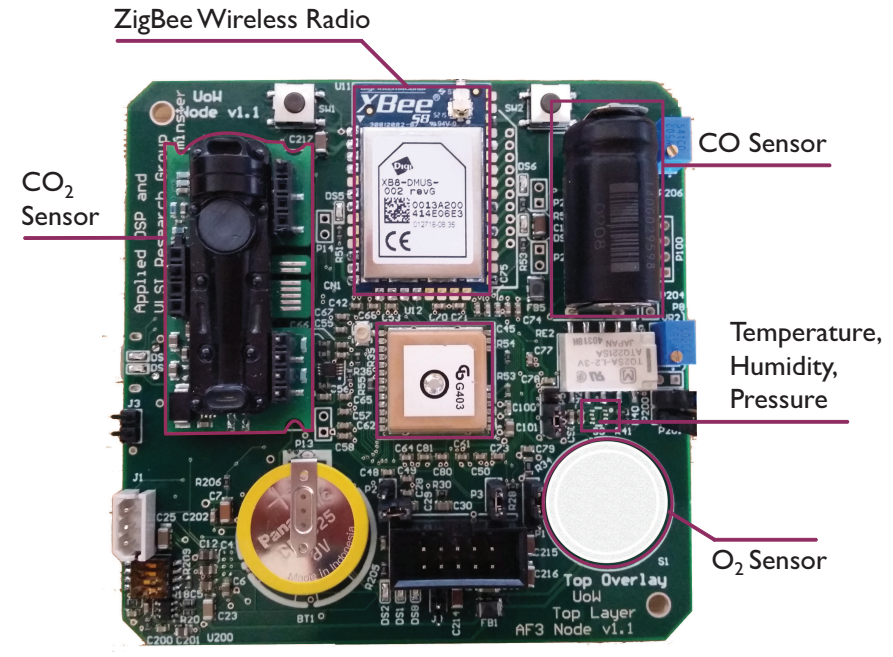

Fig. 1. The top side of the double-sided ground sensor node PCB, where the radio module and sensors are located, since they have to be exposed to the air in order to take measurements. The ground sensor node PCB was designed to fit easily into cylindrical shaped housings shown in Figure 2. The bottom side hosts the CPU, energy management circuit, I2C level converter, USB to UART converter and battery.

fire detection and fighting solution and to develop a complex integrated emergency response system. As a part of the AF3 emergency response system, a Low Power Wireless Ground Sensor Network (LPWGSN) was developed that provides nearreal-time environmental monitoring capabilities. Specifically, the LPWGSN forms part of the project's ground monitoring technologies which deals with the early detection aspect as well as monitoring fire progress. This article details the LPWGSN and its components and gives the results obtained from one of the forest fire trials the network nodes were tested. In comparison to the works from the open literature the individual nodes of the LPWGSN employ a wider set of sensors as well as energy harvesting, signal processing, transmission and reception components for an enhanced network power autonomy as well as maximum area coverage with lower overall cost which has been tested in a real-life firefighting trial. The WSN described herein is built totally from the ground up to target low power as well as providing capabilities to measure key gases enabling a more detailed assessment of the fire environment.

\section{LOW POWER WIRELESS GROUND SENSOR NETWORK NODES}

The ground sensor nodes of the LPWGSN are equipped with various environmental sensors including temperature, humidity, barometric pressure, oxygen, carbon monoxide, carbon dioxide sensors. Furthermore, the nodes contain a Central Processing Unit (CPU), battery, energy managing and harvesting circuit, radio transceiver. The physical location of the nodes are known as they provide location data on first start-up via a Global Positioning System (GPS) module on-board. Each node ID is associated with its geographical coordinates and saved in a database. The sensor nodes have two operating modes
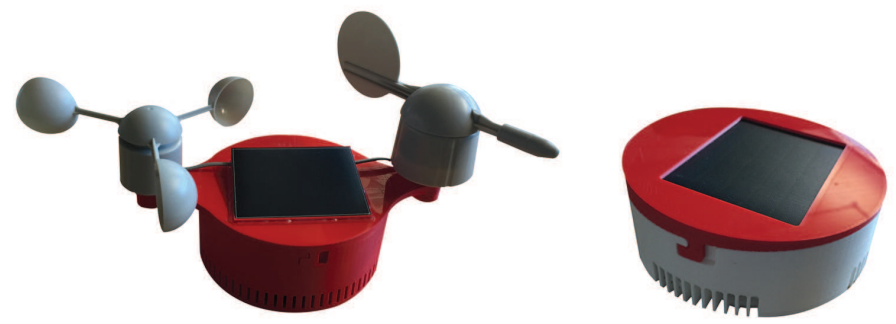

Fig. 2. The housings designed for the Master Node (on the left) and the Sub Node (on the right). Both nodes are equipped with a solar panel that sits on top of the housing to enable energy harvesting. As can be seen the master node has wind velocity and direction measurement instruments.

which are normal and high alert mode. In order to comply with low power consumption requirements in normal mode, the sensor readings are taken less frequently than in high alert mode. In the ground sensor nodes a timer is responsible for the regular reading of all environmental sensors. The sensor reading period is configurable. This is also a function of the battery level such that at critical levels only the most important sensors are sampled and the interval between transmissions is appropriately configured. In a high alert level scenario the sensor reading period is shortened in order to provide data readings more frequently. Configurable thresholds are set for temperature, oxygen, carbon-monoxide, carbon dioxide. If one or multiple sensor reading values are outside the normal threshold level, the node will collect all sensor readings and transfer the data to the Master Node. Figure 1 shows how the sensors and radio module are distributed across the top-side of the sensor node.

Nodes make use of intelligent ZigBee mesh networking (with a XBEE-Digimesh radio transceiver module) enabling network scalability in covering large forest terrain. The ground sensor nodes operate in clusters composed of Master and Sub Nodes. Master nodes act as gateways allowing upload of data to a remote server. Built in redundancy ensures that data collected by sub nodes is uploaded to nearest functional Master. Telemetry data between sub nodes and master node includes all sensor readings, battery level, geographical position, etc.

\section{A. Types of Ground Sensor Nodes}

The two classes of nodes separated by their role in the network are detailed below:

2.1 Sub Node: Sub nodes can measure all primary environmental data such as Temperature, Atmospheric/Barometric Pressure, Relative Humidity, Oxygen, Carbon Dioxide and Carbon Monoxide. In addition to the on-board GNSS module for pinpointing incidents, the sub nodes are equipped with a solar panel enabling energy harvesting.

2.2 Master Node: Master nodes have a GSM/GPRS/3G module (with a WiFi backup) to establish a link with the AF3 Ingestion Server via GPRS connectivity. Master nodes provide wind speed and direction information (via the use of wind velocity and direction measurement instruments) in addition to the base parameters of the sub nodes. The on-board MicroSD 
is for local data storage. Master nodes will request sensor data from all surrounding sensor nodes and alerts and relay all the available data to the AF3 Ingestion Server at the firefighting command center for further processing. Figure 2 shows the housings designed for the master node and the sub node.

\section{B. System Description}

The block diagram of a ground sensor node is given in Figure 3. All of the sensors and the processing components used within the double-sided PCB (the two sides will be called 'Bottom-side' and 'Top-side') are listed in this figure. The bottom side of the ground sensor node hosts the CPU, energy management circuit, I2C level converter, USB to UART converter and battery. This way the sensitive elements of the sensor node are protected from outward exposure. On the top side, the radio module and sensors are placed (as can be seen in Figure 1), since the sensors have to be exposed to the air in order to take measurements. The LTC3586 power management circuit manages the charging of the battery via USB cable and solar panel. This IC outputs three different regulated voltage levels with $99 \%$ efficiency. In addition, it is possible to turn on/off selected power rails, power down peripheries in order to save energy. The USB connector gives access to a serial terminal for debugging via the FT232BL chip on the UART2 port. The GPS module with integrated patch antenna is connected to the UART0 port and it is powered from a switched $3.3 \mathrm{~V}$ line. Therefore, it can be powered down completely in order to save energy. The Luminox O2 sensor is on UART3 and it is powered by the $5 \mathrm{~V}$ switched power supply. The EE893 CO2 sensor is on $\mathrm{I} 2 \mathrm{C} 0$ port and it is necessary to use a voltage converter IC from 3.3 to $5 \mathrm{~V}$ for this particular sensor. The BME280 temperature, humidity and barometric pressure sensor is on the $\mathrm{I} 2 \mathrm{C}$ communication line. The TGS5342 CO sensor is utilized by using an internal analogue circuitry and they are fed from a fixed $3.3 \mathrm{~V}$ power line. These sensors are on all the time and any sudden increase or passing a set threshold level in $\mathrm{CO}$ level or temperature level will trigger a software interrupt. On the board an SPI EEPROM is implemented for storing samples along their time stamps for further processing and data transmission to the master node. The EEPROM retains the data content in case of a total shut down due to lack of power. The RTC clock with a coin battery and LP oscillator are utilised for timing, initial synchronization is achieved via the GPS module or master node. The $50 \mathrm{MHz}$ Oscillator is for clock generation to the PLL. The JTAG pins are for programming and debugging.

\section{Deployment Strategy}

Nodes are deployed in a star topology with a master node served by several sub nodes forming a node cluster. Multiple such clusters will be deployed with overlap in the communication range to provide redundancy. In the case of master node failure in a cluster, the cluster's sub nodes should still be able to convey their data to the nearest live master node for upload to the Server.

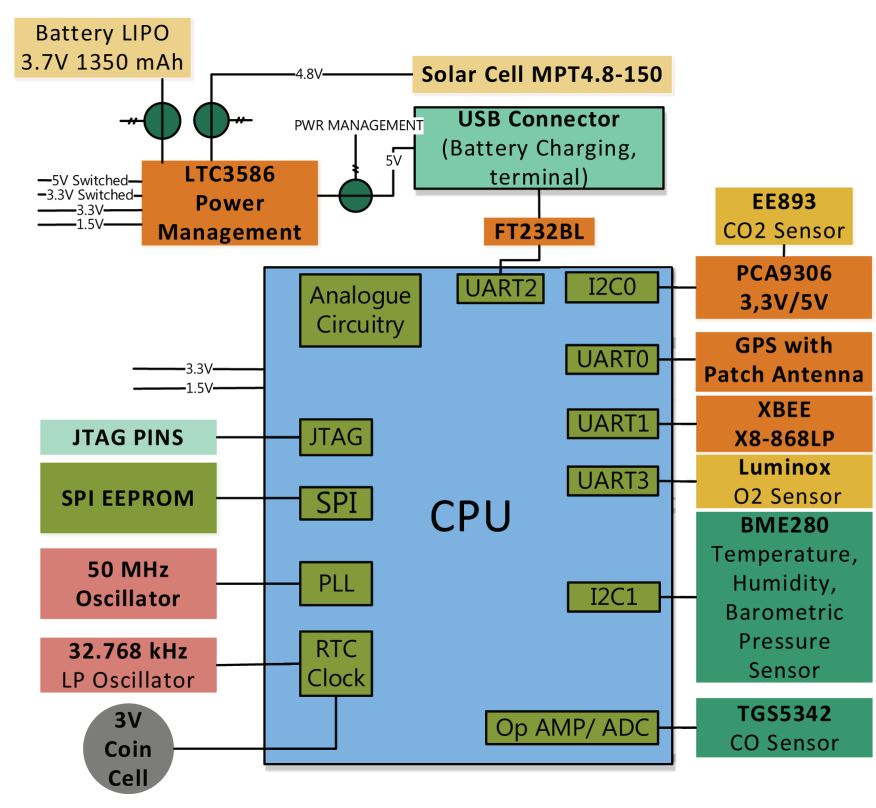

Fig. 3. Block Diagram of Ground Sensor Nodes listing all the components used designing the PCB.

\section{REAL FIREFIGHTING TRIALS}

The LPWGSN and the functionality of its nodes were tested in a real-life firefighting trial held in Greece in May 2016 under the AF3 project. A total of six nodes were available for deployment comprised of one Master Node responsible for the relaying of captured data to the AF3 Ingestion Server and five Sub Nodes solely responsible for monitoring of environmental parameters using their array of on-board sensors. As the nodes were battery powered in addition to having on-board solar cells for power harvesting, no addition external power sources were necessary on the trials. As a result, once the nodes were deployed no manual intervention was necessary. The only requirement was a means of communication with the ingestion server by the Master Node and this was satisfied by the availability of the local GSM network. The performance of the nodes and in turn the overall wireless sensor network was gauged by the performance of various individual aspects of the nodes by themselves and then as a whole. As such this section is divided into various segments, each evaluating these aspects and how they compared against the intended behaviour.

3.1 GPS Test: In order to simplify deployment as well as enable geo-tagging of potential incidents, the nodes were equipped with on-board GPS functionality. After deployment, the locations transmitted by the nodes was analyzed. The locations were found to be accurate to within a few meters as expected. Considering the eventual use case where nodes would expect to be deployed spaced 50/100m apart, this result was acceptable.

3.2 GSM Test: GSM capability is built into the Master Node only and this enables it to communicate with the outside world, thereby allowing it upload all gathered data to the AF3 ingestion server. Initially there were slight issues in 


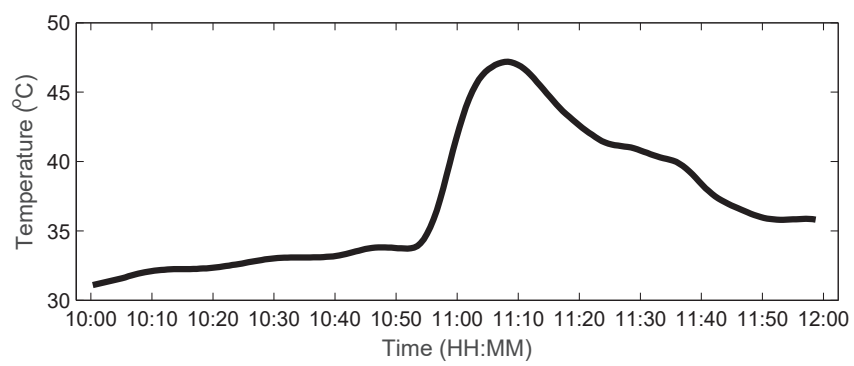

(a)

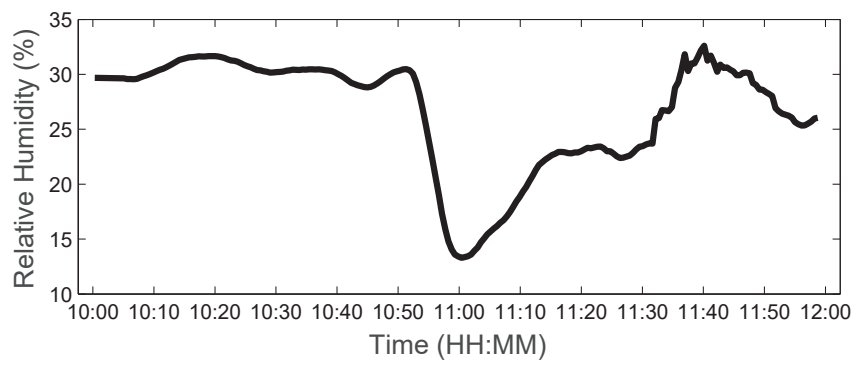

(b)

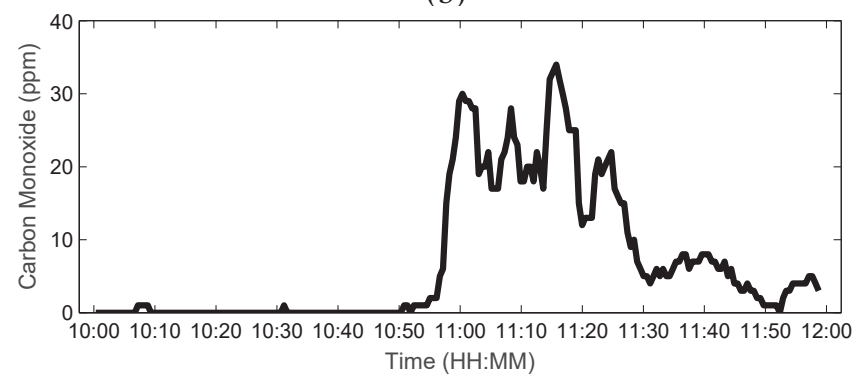

(c)

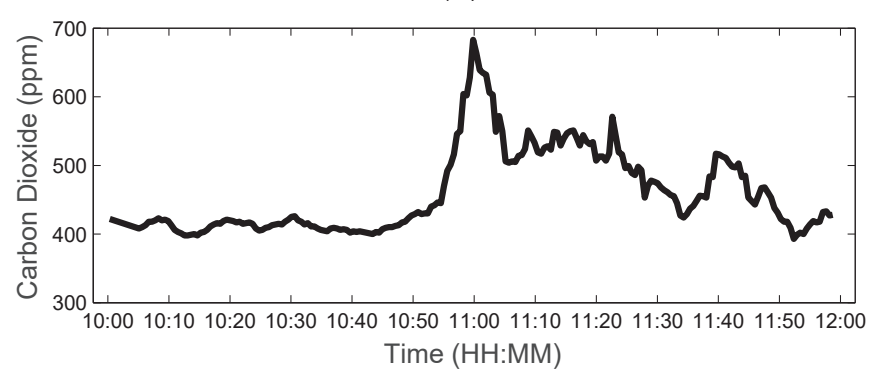

(d)

Fig. 4. Sample data taken from one of the nodes that in particular picked up a significant variation signifying its closer proximity to the fire. Here (a) Temperature (b) Humidity (c) $\mathrm{CO}$ (d) $\mathrm{CO} 2$ information collected from the same node during start and the extinguishing of the fire.

connecting with the server due to poor network coverage in the area. However, once the connection was established data was successfully uploaded.

3.3 Overall Network Functionality: The overall LPWGSN performed as expected and the results were in line with the tests carried out in the labs at our university. Data from each sensor in the network was successfully sent to the Master Node which then uploaded this data to the ingestion server. The sample upload frequency was set to every 40 seconds. All sensors on-board the nodes were responsive throughout the entirety of the trials and the data collected supports this. No

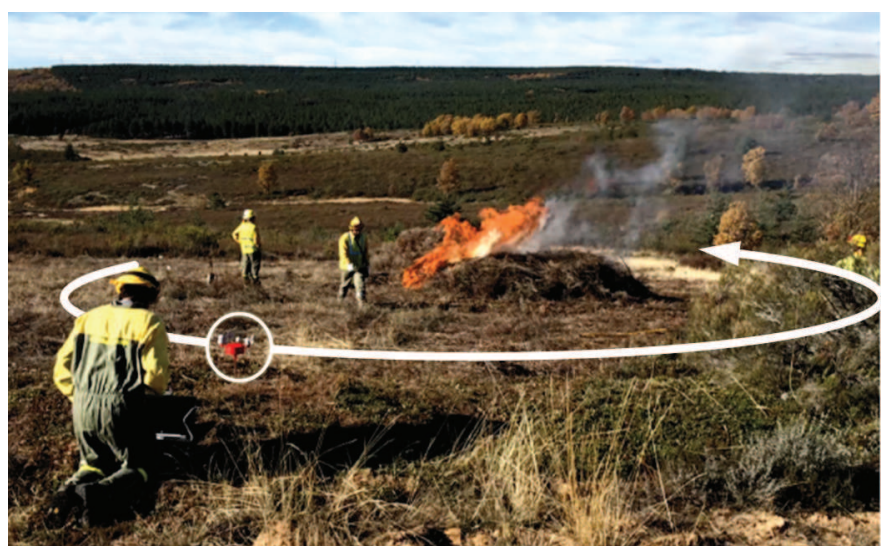

Fig. 5. The nodes are distributed in a circular fashion around the location of the fire. The white circle highlights one of the nodes

major anomalies were seen in the data. Figure 4.a presents the temperature data taken from one of the nodes that in particular picked up a significant temperature variation signifying its closer proximity to the fire. The curve also represents the extinguishing of the fire as the temperatures settle back to pre-fire levels. While not as significant, the other nodes also appear to display change in their temperature curves. On the other hand the relative humidity for the very same node (Figure 4.b) shows good correlation with the temperature data. Unlike temperature, there has been somewhat a significant activity in the other nodes indicative of their proximity to the water activity.

One other significant indicator was the results of the Carbon Monoxide and Carbon Dioxide data. Rapid rise in carbon monoxide and dioxide levels can signal a flaming fire in progress. From the graphs in Figure 4.c and Figure 4.d it can be seen that the sudden rise in $\mathrm{CO} 2$ and $\mathrm{CO}$ coincides with the time the fire begins as seen from the previous sets of figures. However here, the graphs constitutes a multitude of spikes and this is perhaps attributed to $\mathrm{CO} 2$ rapidly dispersing in the atmosphere helped by the windy conditions during the trials. Once again, the nodes nearer the fire exhibit the highest level of change while other not so much. Change in the other parameters, namely atmospheric pressure and oxygen did not show any significant change. This may be due to the small scale of the fire resulting in a low impact on the environment and in turn leading to minimal fluctuations.

While overall WSNs face a challenge in covering large expanses of remote forested terrain due to their reliance on the communication networks deployed in nearby settlements to offload their data from the network to a server for further processing/analysis, our design decisions are a step towards tackling this issue. The use of a reasonably ranged communication protocol together with mesh functionality ensures that data can be reliably routed to any part of the network. The use of a multi data sink (via Master Nodes) network increases the likelihood of being able to offload network data at the edge locations. 


\section{CONCLUSION AND FUTURE WORK}

In this article a low-power wireless sensor node design was detailed within a Low Power Wireless Ground Sensor Network (LPWGSN). The LPWGSN is developed as a part of the Advanced Fire Fighting (AF3) Project that operates under the European Unions Research and Innovation funding programme. The designed nodes were tested during a reallife firefighting trial in Greece where the overall result of the LPWGSN were positive and encouraging. The WSN was able to successfully capture environmental parameters and upload the data to the AF3 Ingestion Server for server-side post processing. The nodes were fully functional throughout both the trials and even withstood the extinguishing activity in the pellet and water drop tests. Due to the ease of use and robustness, our network was also used to monitor the environment during the pellet drop tests as well as collect wind data to help validate one of our partners wind models. This is a testament to the flexible nature of our system and is indicative of the usability of our nodes in various non-critical applications outside of the scope presented here, for example in agriculture, indoor climate control via heating ventilation and air conditioning, urban air quality monitoring. While a total of six nodes were deployed in the initial trials, the network is capable of easily being scaled up by the addition of new nodes as dictated by the requirements of the application. New nodes are automatically integrated into the system as they are detected. Approaching the final trials in Israel under the AF3 project, we hope to further improve upon our systems efficiency and form factor and these results will be presented in a future publication.

\section{ACKNOWLEDGEMENT}

This work has been developed as part of the Advanced Forest Fire Fighting (AF3) project (Grant Agreement no 607276), a collaborative project funded under the Framework 7 programme by the European Commission within the theme SEC-2013.4.1-6 Preparedness for and management of large scale forest fires.

\section{REFERENCES}

[1] J. K. Balch, B. A. Bradley, J. T. Abatzoglou, R. C. Nagy, E. J. Fusco and A. L. Mahood, "Human-started wildfires expand the fire niche across the United States," PNAS, pp. 1-6, 2017.

[2] S. Byungrak, H. Yong-sork and K. Jung-Gyu, "A Design and Implementation of Forest-Fires Surveillance," IJCSNS International Journal of Computer Science and Network Security, pp. 124-130, 2006.

[3] L. Yanjun, W. Zhi and S. Yeqiong, "Wireless Sensor Network Design for Wildfire Monitoring," in Proceedings of the 6th World Congress on Intelligent Control, Dalian, 2006.

[4] A. Molina-Pico, D. Cuesta-Frau, A. Araujo, J. Alejandre and A. Rozas, "Forest Monitoring and Wildland Early Fire Detection by a Hierarchical Wireless Sensor Network," Journal of Sensors, vol. 2016, pp. 1-8, 2016.

[5] D. M. Doolin, N. Sitar, "Wireless sensors for wildfire monitoring," Proc. Of SPIE Symposium on Smart Structures and Materials, San Diego, pp. 477-484, 2005. 